\title{
Coherent defect-assisted multiphonon intraband carrier relaxation in semiconductor quantum dots
}

\author{
A.N. Poddubny ${ }^{1}$ and S.V. Goupalov ${ }^{1,2}$ \\ 1 A.F. Ioffe Physico-Technical Institute, Russian Academy of Sciences, St. Petersburg 194021, Russia \\ 2 Department of Physics, University of Utah, Salt Lake City, UT 84112, USA
}

\begin{abstract}
A new defect-assisted mechanism of multiphonon intraband carrier relaxation in semiconductor quantum dots, where the carrier is found in a coherent superposition of the initial, final, and defect states, is proposed. It is shown that this mechanism is capable of explaining the observed trends in temperature dependences of the intraband relaxation rates for PbSe and CdSe colloidal nanocrystal quantum dots.

PACS numbers: 71.38.-k,78.67.Hc
\end{abstract}

\section{INTRODUCTION}

Recent experimental studies of PbSe nanocrystals (NCs) have revealed an unexpectedly fast intraband relaxation in semiconductor quantum dots (QDs) with energy separation between adjacent electron (or hole) levels exceeding by far the optical phonon energies 1,2. A pronounced temperature dependence of the observed relaxation rates 2 suggests that underlying mechanism should involve multiphonon transitions while Auger-like relaxation processes 3 are ruled out due to a nearly symmetric energy spectra in the conduction and valence bands of PbSe.

The multiphonon transitions between otherwise orthogonal quantized electronic states in a QD may occur due to nonadiabaticity of the electron-phonon system [4,5,6,7. A model study performed in conjunction with the experiments on $\mathrm{PbSe} \mathrm{NCs}$ revealed that, in order for this mechanism to be responsible for the experimentally observed temperature dependence of the relaxation rate, the electron-phonon coupling should be stronger than one would expect 7. However, the study of detailed models of electron-phonon interaction in quantum dots (upon which the judgement about the coupling strength is based) has always been a weak point in approaches to energy relaxation 8 and Raman 9 processes. In case of PbSe NCs the situation is further obscured by the fact that the widely accepted model of their electronic structure 10 which would be natural to pick for an estimate of electron-phonon coupling strength fails to describe absorption spectra of $\mathrm{PbSe} \mathrm{NCs} 1$ and is therefore not quite reliable. Thus, instead of seeking to either confirm or rule out this mechanism we will concentrate on examining other possibilities for the fast energy relaxation.

As it has been proposed for epitaxially grown QDs 11, such possibilities can be provided by a localized state of an impurity or defect close to the QD surface. In case of the experiment of Ref. 1 such states can correspond to deep impurities in the silica host surrounding PbSe NCs occurring in an appreciable proximity to the NC surface. Otherwise they can be due to surface states. An electron trapped to the impurity is strongly localized which assures its strong coupling to local lattice vibrations. One can then consider a relaxation process involving the interior QD levels as the initial (2) and final (0) states, and the state at the impurity as an intermediate (1) state as shown in Fig. 1. Although processes of this type have been theoretically investigated 8, 11, 12, all these studies considered the relaxation as a two step process, so the relaxation time $\tau \equiv \tau_{2 \rightarrow 0}=\tau_{2 \rightarrow 1}+\tau_{1 \rightarrow 0}$. But if $\tau_{1 \rightarrow 0}$ is shorter than the phonon lifetime in state 1 then the transition $2 \rightarrow 0$ must be treated as a coherent quantum mechanical process. Such process represents a new mechanism of phonon-assisted carrier relaxation in QDs and its studying is the scope of the present work. Similar processes have been considered for capture or generation of electron-hole pairs by impurities in bulk semiconductors 13

Another system where the proposed mechanism can play the key role is provided by CdSe colloidal nanocrystals. In the recent experiments on such quantum dots the fast intraband relaxation of electrons has been observed in the absence of the holes 15 . The electrons were injected into the quantum dots instead of being optically generated, and the pump-probe measurements were subsequently carried out 15 . These experiments showed that intraband relaxation times vary significantly with surface modification suggesting involvement of a surface state such as the empty $s$ - state of the surface $\mathrm{Cd}^{2+}$ atoms 15 . A faster relaxation rate was observed for lower temperatures 15 though more thorough studies of the temperature dependences of the relaxation rate in CdSe colloidal nanocrystals are desirable.

It has been noticed 7 that multiphonon relaxation processes are analogous to tunneling problems in electron transport utilizing the tunneling hamiltonian. It turns out that the relaxation mechanism proposed in this paper is analogous to the problem of electron tunneling through a potential barrier placed between two leads and containing localized electronic states coupled to phonons and resonant with the energy of the tunneling electron. The latter problem was studied by Glazman and Shekhter 16 . There is, however, a substantial difference between the two problems due to the descrete energy spectrum for carriers confined in a QD. Indeed, in the problem of electron tunneling there is a continuum of both initial and 
final states in the leads. Thus, this problem has a characteristic time related to the elastic tunneling of the electron from the localized state to either of the leads and determining the width of the resonance. The rate of the phonon-assisted tunneling can be then calculated using some generalization of the Fermi golden rule with the $\delta$-function accounting for the energy conservation being integrated out while summing over the continuum of final electronic states. In our problem the initial and final electronic states are descrete. However, the spectrum of the entire system "electron plus phonons" is continuous and the scheme of calculation of the transition rate proposed in Ref. 16 can still be applied, although some changes should be introduced. First, the width of the resonance will be determined by the lifetime of phonons in the intermediate state. Second, the phonon density of states will enter the expression for the transition rate. It turns out that these changes can be more naturally incorporated using a more traditional approach based on calculation of overlap integrals of eigenfunctions belonging to shifted parabolic potentials (see e.g. 12). Another advantage of this method is that it allows one to make some qualitative conclusions without having done actual calculations.

In this paper the proposed mechanism of carrier relaxation will be studied in detail for a model QD system while discussions of its relevance to particular realizations of QDs are left for future studies. The rest of the paper is organized as follows. In Sec. II we formulate the problem in a form most convenient for comparison with the work by Glazman and Shekhter 16 and with the case of nonadiabaticity-induced phonon-assisted transitions between intrinsic QD states 7. The relaxation rate is expressed through a correlation function which should be calculated taking into account phonon decay. In Sec. III we devise an approximation allowing one to avoid evaluation of this correlation function. The lifetime of the intermediate state in the relaxation process is derived in a consistent way. In Sec. IV results of Sec. III are implemented into calculation of the relaxation rate using direct evaluation of the overlap integrals. Dependences of the relaxation rate on temperature and electron-phonon coupling strength are discussed in Sec. $\mathrm{V}$

\section{MODEL}

The Hamiltonian describing multiphonon nonradiative transitions between three electronic levels with unperturbed energies $\varepsilon_{0}, \varepsilon_{1}, \varepsilon_{2}$ can be represented as $H=$ $H_{0}+V$, where $H_{0}$ is the phonon Hamiltonian describing local vibrations in the electronic state with the energy $\varepsilon_{2}$,

$$
H_{0}=\omega_{0} b^{\dagger} b
$$

and

$$
\begin{array}{r}
V=\varepsilon_{0} a^{\dagger} a+\left[\varepsilon_{1}+\Delta\left(b^{\dagger}+b\right)\right] c^{\dagger} c+\varepsilon_{2} d^{\dagger} d+ \\
\left(B_{01} a^{\dagger} c+\text { H.c. }\right)+\left(A_{12} c^{\dagger} d+\text { H.c. }\right) .
\end{array}
$$

Here $a, c, d$ are the electronic annihilation operators of the electronic states with the energies $\varepsilon_{0}, \varepsilon_{1}, \varepsilon_{2}$, respectively, and $b$ are the phonon annihilation operators. We will first neglect the phonon decay and introduce it later on. Parameter $\Delta$ determines the shift between the minima of the vibrational potentials (corresponding to local vibrations and associated with different electronic states) whose dependence on the configuration coordinate is sketched in Fig. 1. $A_{12}$ and $B_{01}$ describe electronic tunneling between the QD states and impurity level (see Ref. 11 for details).

We write the electronic wave function as

$$
\hat{\Psi}(t)=\left[\hat{u}(t) c^{\dagger}+\hat{v}(t) d^{\dagger}+\hat{w}(t) a^{\dagger}\right]|0\rangle,
$$

where $|0\rangle$ is the electronic vacuum and $\hat{u}(t), \hat{v}(t), \hat{w}(t)$ are the operators acting on the phonon subsystem. The wave function (2) satisfies the Schrödinger equation in the interaction representation with respect to the Hamiltonian $H_{0}$

$$
i \frac{\partial \hat{\Psi}(t)}{\partial t}=\hat{V}(t) \hat{\Psi}(t)
$$

with initial conditions

$$
\hat{u}(t=0)=\hat{w}(t=0)=0, \quad \hat{v}(t=0)=1 .
$$

Substituting Eqs. (12)-(2) into Eq. (3), we obtain the following system of equations

$$
\begin{gathered}
i \frac{\partial \hat{u}}{\partial t}=\left[\varepsilon_{1}+\hat{\mathcal{D}}(t)\right] \hat{u}(t)+B_{10} \hat{w}(t)+A_{12} \hat{v}(t), \\
i \frac{\partial \hat{w}}{\partial t}=\varepsilon_{0} \hat{w}(t)+B_{01} \hat{u}(t) \\
i \frac{\partial \hat{v}}{\partial t}=\varepsilon_{2} \hat{v}(t)+A_{21} \hat{u}(t)
\end{gathered}
$$

where

$$
\hat{\mathcal{D}}(t)=\Delta\left(b^{\dagger} e^{i \omega_{0} t}+b e^{-i \omega_{0} t}\right) .
$$

We will follow Refs. 7, 16 and introduce the auxiliary operator $\hat{u}_{0}(t)$ as the solution of the following Cauchy problem:

$$
i \frac{\partial \hat{u}_{0}}{\partial t}=\hat{\mathcal{D}}(t) \hat{u}_{0}, \quad \hat{u}_{0}(t=0)=1 .
$$

In an explicit form it reads

$$
\begin{array}{r}
\hat{u}_{0}=\exp \left\{\frac{\Delta}{\omega_{0}}\left[b\left(e^{-i \omega_{0} t}-1\right)-H . c .\right]+\right. \\
\left.\left(\frac{\Delta}{\omega_{0}}\right)^{2}\left(i \omega_{0} t-i \sin \omega_{0} t\right)\right\} .
\end{array}
$$


With the help of this operator Eq. (5) can be written as

$$
i \frac{\partial}{\partial t}\left(\hat{u}_{0}^{-1} \hat{u}\right)=\varepsilon_{1} \hat{u}_{0}^{-1} \hat{u}+\hat{u}_{0}^{-1}\left[B_{10} \hat{w}(t)+A_{12} \hat{v}(t)\right] .
$$

Using Eqs. (6), (8) the operator $\hat{w}(t)$ can be calculated in the lowest order in the tunneling matrix elements $B_{01}$, $A_{12}$ as

$$
\begin{array}{r}
\hat{w}(t)=-B_{01} A_{12} \int_{0}^{t} d t_{0} \int_{0}^{t_{0}} d t_{1} e^{i\left(t_{0}-t\right) \varepsilon_{0}} e^{i\left(t_{1}-t_{0}\right) \varepsilon_{1}} \times \\
e^{-i \varepsilon_{2} t_{1}} \hat{u}_{0}\left(t_{0}\right) \hat{u}_{0}^{-1}\left(t_{1}\right) .
\end{array}
$$

The probability of the electron transition is given by

$$
W_{2 \rightarrow 0}=\frac{1}{\tau_{2 \rightarrow 0}}=\lim _{t \rightarrow \infty} \frac{\left\langle\hat{w}^{\dagger}(t) \hat{w}(t)\right\rangle}{t} .
$$

The average here is taken over the initial states of the phonon subsystem at $t=0$. It is assumed that these states are equilibrium states. We also have taken into account that, at $t=0$, there is no interaction-induced re-normalization of the phonon states since the impurity electronic level is empty. Substituting Eq. (9) into Eq. (10) we obtain

$$
\begin{aligned}
W_{2 \rightarrow 0} & =\lim _{t \rightarrow \infty} Z / t \int_{0}^{t} d t_{0} \int_{0}^{t_{0}} d t_{1} \int_{0}^{t} d t_{0}^{\prime} \int_{0}^{t_{0}^{\prime}} d t_{1}^{\prime} e^{-i\left(t_{0}^{\prime}-t_{0}\right) \varepsilon_{0}} \times \\
& e^{i\left(t_{1}-t_{0}-t_{1}^{\prime}+t_{0}^{\prime}\right) \varepsilon_{1}} e^{i \varepsilon_{2}\left(t_{1}^{\prime}-t_{1}\right)} U\left(t_{0}, t_{1}, t_{0}^{\prime}, t_{1}^{\prime}\right),
\end{aligned}
$$

where we have introduced the correlation function

$$
U\left(t_{0}, t_{1}, t_{0}^{\prime}, t_{1}^{\prime}\right)=\left\langle\hat{u}_{0}\left(t_{1}^{\prime}\right) \hat{u}_{0}^{-1}\left(t_{0}^{\prime}\right) \hat{u}_{0}\left(t_{0}\right) \hat{u}_{0}^{-1}\left(t_{1}\right)\right\rangle,
$$

and

$$
Z=\left|B_{01}\right|^{2}\left|A_{12}\right|^{2} .
$$

The correlation function (12) is analogous to the one which appeared in the problem studied by Glazman and Shekhter 16. It was evaluated in Ref. 16 neglecting phonon decay. In our case phonon decay should be taken into account as it determines the width of the resonance corresponding to the intermediate state in the relaxation problem under study. However, the problem of evaluating the correlation function (12) taking the phonon decay into account is very difficult. Therefore, in the next section we devise an approximation which allows one to account for phonon decay without evaluating correlation function (12).

\section{THE WIDTH OF THE RESONANCE}

In order to understand the origin of the width of the resonance corresponding to the intermediate state in our

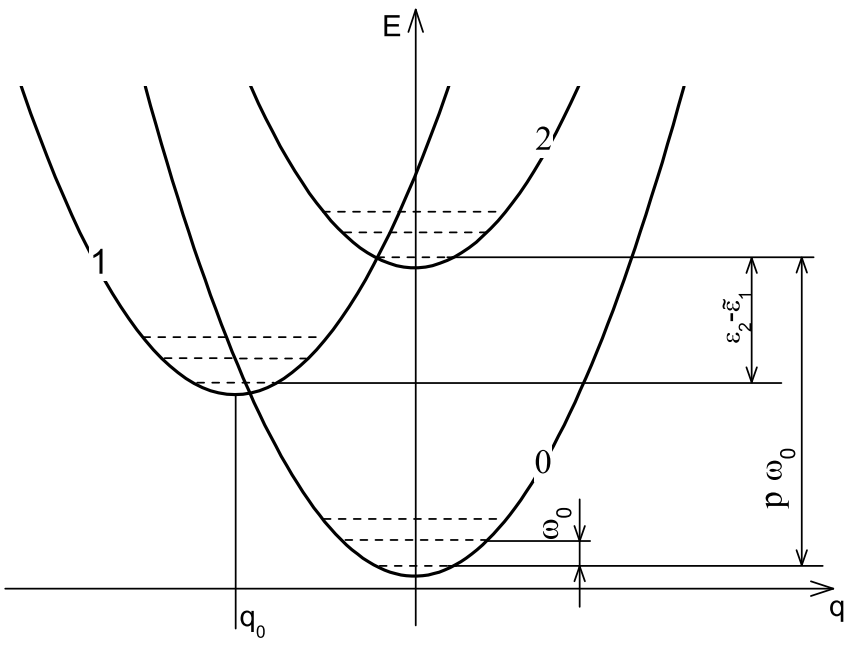

FIG. 1: Energy diagram representing adiabatic potentials for the quantum dot states (0 and 2) and the impurity level (1).

relaxation problem we will consider an auxiliary problem which will help us to apply the concept of quasistationary states to the states of the system "electron localized at the impurity plus phonons". More precisely, let us set $B_{10}=A_{12}=0$ and consider the electron localized at the impurity and coupled to phonons. One can introduce the retarded Green's function of the electron as

$$
G_{R}(t)=-i\left\langle\left\langle 0\left|\tilde{c}(t) \tilde{c}^{\dagger}(0)+\tilde{c}^{\dagger}(0) \tilde{c}(t)\right| 0\right\rangle\right\rangle \theta(t),
$$

where $\tilde{c}(t)$ is the electron annihilation operator in the Heisenberg representation and $\langle\langle 0|\ldots| 0\rangle\rangle$ denotes a quantum mechanical average over the electronic vacuum and a statistical average over the phonon degrees of freedom described by the Hamiltonian $H_{0}$. As the electron creation operator $\tilde{c}^{\dagger}(0)$ enters the Green's function at $t=0$, the latter reflects what happens to the electron when it is suddenly brought to the impurity site from a remote location (where it does not experience any coupling or entangling to phonons) at $t=0$. Therefore, this auxiliary problem is essentially equivalent to the initial relaxation problem. The only difference is that, as $A_{12}=B_{01}=0$, the auxiliary problem does not describe dynamics of the electron tunneling to the impurity site. The Green's function (13) can be written in terms of the operator $\hat{u}_{0} 17$

$$
G_{R}(t)=-i e^{-i \varepsilon_{1} t}\left\langle\hat{u}_{0}(t)\right\rangle \theta(t) .
$$

The spectral function defined as 17

$$
A(\omega)=-2 \Im \int_{-\infty}^{\infty} d t G_{R}(t) e^{i \omega t}
$$

consists of a series of $\delta$-functional peaks separated by the phonon energy $\omega_{0}$. Now, if there are processes leading to the phonon decay then the expression for $\left\langle\hat{u}_{0}(t)\right\rangle$ be- 
comes 18

$$
\begin{array}{r}
\left\langle\hat{u}_{0}(t)\right\rangle=\exp \left\{-\Delta^{2}\left[\frac { \overline { n } _ { 0 } + 1 } { ( \gamma ( \omega _ { 0 } ) + i \omega _ { 0 } ) ^ { 2 } } \left(e^{-\left(\gamma\left(\omega_{0}\right)+i \omega_{0}\right.}\right.\right.\right. \\
+\frac{\bar{n}_{0}}{\left(\gamma\left(\omega_{0}\right)-i \omega_{0}\right)^{2}}\left(e^{-\left(\gamma\left(\omega_{0}\right)-i \omega_{0}\right) t}-1\right)+ \\
\left.\left.t \frac{\gamma(0)\left(2 \bar{n}_{0}+1\right)-i \omega_{0}}{[\gamma(0)]^{2}+\omega_{0}^{2}}\right]\right\} .
\end{array}
$$

Here $\bar{n}_{0} \equiv \bar{n}\left(\omega_{0}\right)$ stays for the Planckian factor, $\gamma(\omega)$ is the frequency-dependent phonon decay rate defined through the imaginary part of the phonon polarization operator 18. At zero frequency $\gamma(0)=0$ (which reflects the fact that the zero phonon absorption linewidth is not affected by the phonon decay) 18, 19. Thus, when (16) is substituted into Eqs. (14), (15), each peak of the spectral function, except for the one corresponding to no phonons, becomes broadened. It is natural to expect that it is this kind of broadening which causes the finite width of the resonance associated with the impurity in our relaxation problem.

Assuming $\gamma\left(\omega_{0}\right) \equiv \gamma \ll \omega_{0}$, the shape of each peak of the spectral function can be described analytically. Eq. (16) yields

$$
\begin{array}{r}
\left\langle\hat{u}_{0}(t)\right\rangle=\exp \left\{S\left[e^{-\gamma t}\left[\left(2 \bar{n}_{0}+1\right) \cos \omega_{0} t-i \sin \omega_{0} t\right)\right]+\right. \\
\left.\left.i \omega_{0} t-\left(2 \bar{n}_{0}+1\right)\right]\right\}
\end{array}
$$

where the Huang-Rhys factor $S$ is given by

$$
S=\left(\frac{\Delta}{\omega_{0}}\right)^{2}
$$

Substituting Eqs. (17), (14) into Eq. (15) we obtain

$$
\begin{aligned}
A(\omega)= & \sum_{l=-\infty}^{\infty} A_{l}\left(\omega-\omega_{l}\right), \quad \omega_{l}=\varepsilon_{1}-S \omega_{0}+l \omega_{0}, \\
A_{l}(\omega)= & 2 e^{l \beta \omega_{0} / 2-\left(2 \bar{n}_{0}+1\right) S} \times \\
& \Re \int_{0}^{\infty} d t I_{l}\left[2 S \sqrt{\bar{n}_{0}\left(\bar{n}_{0}+1\right)} \exp (-\gamma t)\right] e^{i \omega t} .
\end{aligned}
$$

Eq. (18) contains the averaged number of phonons, $\bar{n}_{0}$. It is convenient to write out explicitly contributions of the initial states with given numbers of phonons. To that end we will use

$$
I_{l}(x)=\left(\frac{x}{2}\right)^{l} \sum_{k=0}^{\infty}\left(\frac{x}{2}\right)^{2 k} \frac{1}{k !(k+l) !}
$$

and introduce $z=\exp \left(-\beta \omega_{0}\right)$ so that $\bar{n}_{0}=z /(1-z)$. Substituting this into Eq. (18) we obtain

$$
\begin{aligned}
A_{l}(\omega)= & 2 e^{-S} \sum_{k=0}^{\infty} \frac{\gamma(2 k+l)}{\omega^{2}+(2 k+l)^{2} \gamma^{2}} \times \\
& \frac{z^{k}}{(1-z)^{2 k+l}} \exp \left(-\frac{2 S z}{1-z}\right) \frac{S^{2 k+l}}{k !(k+l) !} .
\end{aligned}
$$

This expression can be recast using the generating function for associated Laguerre polynomials 20

$$
\frac{z^{k}}{(1-z)^{2 k+l}} \exp \left(-\frac{2 S z}{1-z}\right)=(1-z) z^{k} \sum_{n=0}^{\infty} z^{n} L_{n}^{2 k+l}(2 S) \text {. }
$$

As a result, Eq. (19) can be recast in the form

$$
A_{l}(\omega)=\left(1-e^{-\beta \omega_{0}}\right) \sum_{m=0}^{\infty} A_{l m}(\omega) e^{-m \beta \omega_{0}}
$$

where

$$
\begin{aligned}
& A_{l m}(\omega)=2 e^{-S} \times \\
& \quad \sum_{k=0}^{m} \frac{(2 k+l) \gamma}{\omega^{2}+(2 k+l)^{2} \gamma^{2}} \frac{S^{2 k+l}}{k !(k+l) !} L_{m-k}^{2 k+l}(2 S) .
\end{aligned}
$$

Each term in the r.h.s. of Eq. (20) corresponds to a transition from the initial state with $m$ phonons into the impurity state with $m+l$ phonons and describes this transition's contribution into the $l$-th peak of the spectral function $A_{l}(\omega)$. The latter is contributed by all processes where exactly $l$ phonons are emitted. Each of the contributions $A_{l m}(\omega)$ consists of $(m+1)$ Lorentzians. The Lorentzians with small $k$ in the r.h.s. of Eq. (21) can have negative weights while the weight of the Lorentzian with $k=m$ is always positive. The latter Lorentzian determins spectral wings of $A_{l m}(\omega)$ and it is natural to associate its width, $\gamma(2 m+l)$, with the linewidth of the transition between the states with $m$ and $m+l$ phonons. From the theory of spectral linewidths it is well known 21 that the spectral width of a line corresponding to a transition between two quasistationary states is given by the sum of their decay rates. As $\gamma$ is the phonon decay rate and we have $m$ phonons in the initial state and $(m+l)$ phonons in the impurity state, we finally arrive at the assignment of the width $\gamma(m+l)$ to the state of the electron at the impurity entangled to $(m+l)$ phonons.

\section{THE TRANSITION RATE}

We have seen that the lifetime of the intermediate state in the relaxation problem under study depends on the number of phonons associated with it. In this case it is more convenient to consider the relaxation process in the framework of an approach based on calculation of overlap integrals between eigenfunctions belonging to shifted parabolic potentials. These potentials for our system are schematically depicted in Fig. 1]. For the quantum dot states the potentials are given by 6

$$
\begin{aligned}
& V_{0}(q)=\frac{M \omega_{0}^{2} q^{2}}{2}+\varepsilon_{0}, \\
& V_{2}(q)=\frac{M \omega_{0}^{2} q^{2}}{2}+\varepsilon_{2} .
\end{aligned}
$$



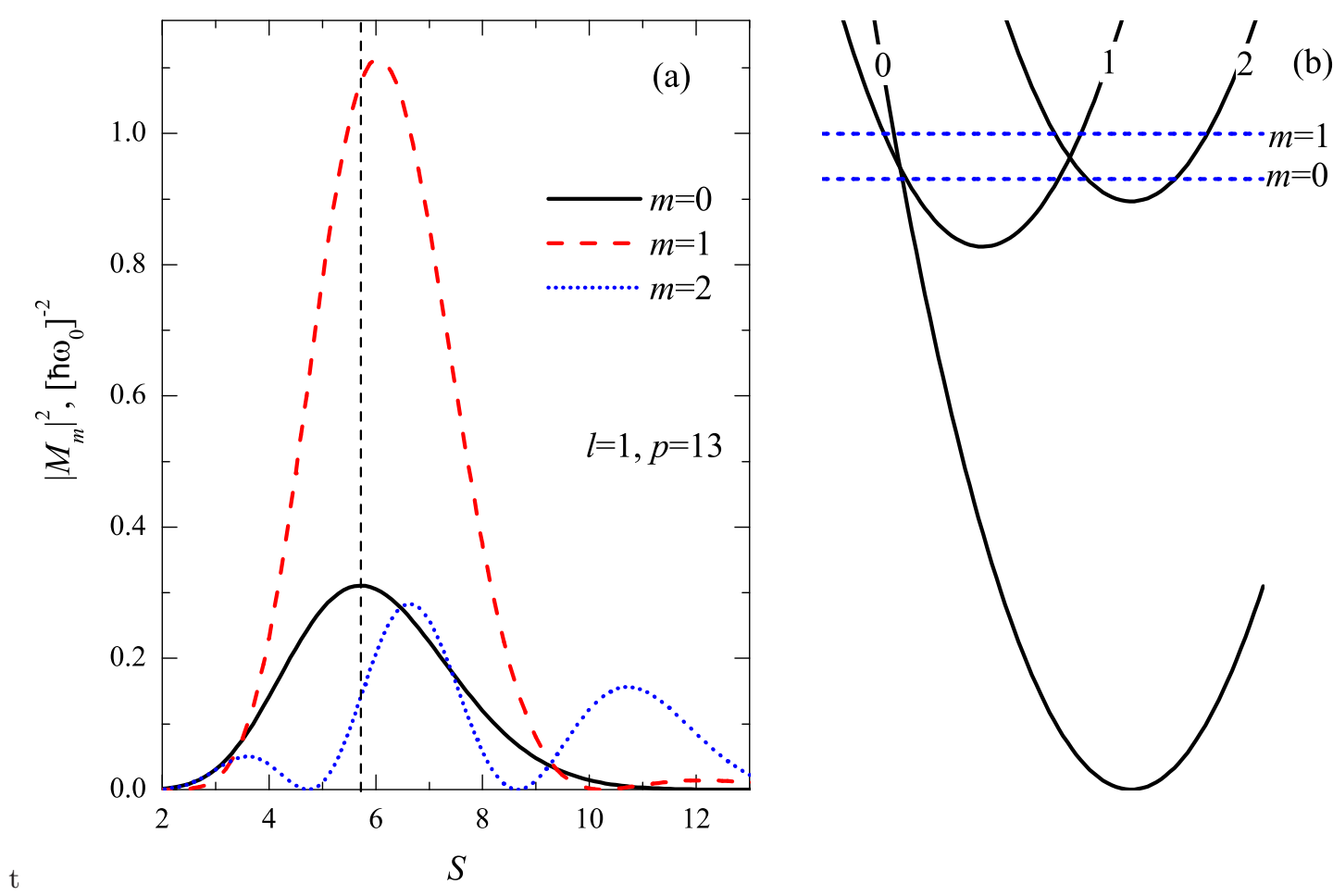

FIG. 2: (a) The dependence of the matrix elements $\left|M_{m}\right|^{2}$ on the Huang-Rhys factor, $S$, calculated for different values of $m=0,1,2,3$ with $l=1, p \omega_{0}=200 \mathrm{meV}, p=13, \gamma=0.2 p s^{-1}$. The vertical dashed line indicates the maximum of the dependence $\left|M_{0}\right|^{2}(S)$ at $S=5.7$. (b) Adiabatic curves, Eqs. (22) - 24), calculated for $S=5.7$. The horizontal lines indicate positions of the energy levels in the potential $V_{2}$ with $m=0$ and $m=1$.

Here $\omega_{0}$ is the frequency of the local vibrational mode, $q$ is the corresponding configurational coordinate, and $M$ is the mass of the impurity's ion. The adiabatic potential for the intermediate state is given by $\underline{6}$

$$
V_{1}(q)=\frac{M \omega_{0}^{2}\left(q+q_{0}\right)^{2}}{2}+\tilde{\varepsilon}_{1},
$$

where

$$
\begin{gathered}
q_{0}=\sqrt{2 S /(M \omega)}, \\
\tilde{\varepsilon}_{1}=\varepsilon_{1}-S \omega_{0} .
\end{gathered}
$$

Let us introduce the eigenfunctions $\psi_{n}^{(2)}(q), \psi_{n}^{(1)}(q)$, and $\psi_{n}^{(0)}(q)$ in the adiabatic potentials $V_{2}(q), V_{1}(q)$, and $V_{0}(q)$, respectively. These are eigenfunctions of the harmonic oscillator satisfying the following relation $\psi_{n}^{(2)}(q)=\psi_{n}^{(1)}\left(q-q_{0}\right)=\psi_{n}^{(0)}(q)$. Then the transition rate $W_{2 \rightarrow 0}$ is given by the Fermi golden rule

$$
W_{2 \rightarrow 0}=\frac{2 \pi Z}{\omega_{0}}\left\langle\left|M_{2 \rightarrow 0}\right|^{2}\right\rangle,
$$

where

$$
\left\langle\left|M_{2 \rightarrow 0}\right|^{2}\right\rangle=\left(1-e^{-\beta \omega_{0}}\right) \sum_{m=0}^{\infty} e^{-\beta m \omega_{0}}\left|M_{m}\right|^{2},
$$

$$
M_{m}=\sum_{n=0}^{\infty} \frac{\left\langle\psi_{m}^{(2)} \mid \psi_{n}^{(1)}\right\rangle\left\langle\psi_{n}^{(1)} \mid \psi_{m+p}^{(2)}\right\rangle}{(m-n) \omega_{0}+\varepsilon_{2}-\varepsilon_{1}+S \omega_{0}+i \gamma n} .
$$

The energy $\delta$-function in Eq. (26) has been integrated out yielding the phonon density of states $\omega_{0}^{-1}$ while the value of $\omega_{0}$ is adjusted to make the number $p=\left(\varepsilon_{2}-\varepsilon_{0}\right) / \omega_{0}$ integer. We also took into account that, according to Sec. III. the width of the intermediate state is given by the number of phonons in that state times the phonon decay rate.

The overlap integrals entering Eq. (28) can be expressed in terms of the generalized Laguerre polynomials 22

$$
\begin{aligned}
&\left\langle\psi_{m}^{(0)} \mid \psi_{n}^{(1)}\right\rangle=(-1)^{(m-n) / 2+|m-n| / 2} \times \\
& e^{-S / 2} \sqrt{\frac{\min (m, n) !}{\max (m, n) !}} \sqrt{S}{ }^{|m-n|} L_{\min (m, n)}^{|m-n|}(S) .
\end{aligned}
$$

The relaxation is most efficient when the energy of the intermediate state is in resonance with that of the initial and final states. We will assume that the resonance condition is always fulfilled and, therefore,

$$
\varepsilon_{2}-\varepsilon_{1}+S \omega_{0}=l \omega_{0}, \quad l \in \mathbb{N} .
$$

In this case the term with $n=l+m$ becomes dominant 
in Eq. (28), and we obtain

$$
M_{m} \approx \frac{-i}{\gamma(l+m)}\left\langle\psi_{m}^{(2)} \mid \psi_{l+m}^{(1)}\right\rangle\left\langle\psi_{l+m}^{(1)} \mid \psi_{m+p}^{(2)}\right\rangle .
$$

\section{RESULTS AND DISCUSSION}

At zero temperature, only the contribution with $m=0$ survives in Eq. (27), and the dependence of the relaxation rate on the strength of the electron-phonon coupling is governed by the dependence of $\left|M_{0}\right|^{2}$ on the Huang-Rhys factor $S$. This dependence is shown in Fig. 2 (a) (solid curve). Both $S$ and $\varepsilon_{1}$ were changed in such a way that the energy difference $\varepsilon_{2}-\varepsilon_{1}+S \omega_{0} \equiv l \omega_{0}$ remained fixed. This is equivalent to a horizontal shift of the adiabatic curve 1 in Fig. 2 (b). As one can see from Fig. 2. (a), the dependence $\left|M_{0}\right|^{2}(S)$ has a maximum yielding the optimal value of the Huang-Rhys factor at which the relaxation is the most efficient. This can be understood using quasiclassical language. According to the quasiclassical theory, the overlap integral of eigenfunctions belonging to different adiabatic potentials is maximum at those energy values where the adiabatic curves intersect 6 . The optimal value of the Huang-Rhys factor corresponds to the situation where both intersection points are close to the energy level with $m=0$, as shown in Fig. 2 (b). This figure also explains why maximum of the curve $\left|M_{1}\right|^{2}(S)$, also shown in Fig. 2, (a), is close to the maximum of $\left|M_{0}\right|^{2}(S)$. As temperature is increased, thermal activation leads to increasing importance of the terms in the r.h.s. of Eq. (27) with $m>0$. In the meantime, relative contribution of the term with $m=0$ to the transition rate decreases due to the factor of $1-\exp \left(-\beta \omega_{0}\right)$ in Eq. (27). Therefore, the character of the temperature dependence of the relaxation rate is determined by the ratio of $\left|M_{0}\right|$

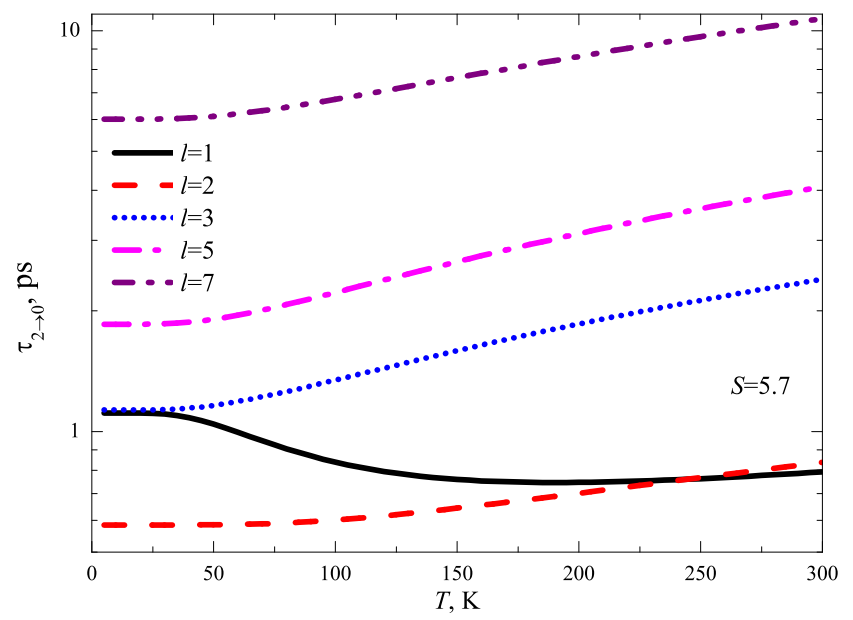

FIG. 3: Temperature dependences of the relaxation time calculated for different values of the impurity level position $(l=1,2,3,5,7)$ with $Z=(2.5)^{4} \mathrm{meV}^{4}, S=5.7$. Other parameters are the same as in Fig. 2 . and $\left|M_{1}\right|$. One can see from Fig. 2. (a) that, for $l=1$ and Huang-Rhys factor close to its optimal value, $\left|M_{1}\right|$ exceeds $\left|M_{0}\right|$, and relaxation time decreases with temperature as shown in Fig. 3 (solid curve). For $l>1$ intersection points would be closer to the level with $m=0$ than to the level with $m=1$ resulting in $\left|M_{0}\right|>\left|M_{1}\right|$. This will lead to increasing temperature dependence of the relaxation time, as shown in Fig. 3.

Therefore, the present mechanism can reproduce both increasing and decreasing temperature dependences of the relaxation time. Those are two types of temperature dependences observed experimentally 2, 15. Note that when temperature sweeps from zero to $300 \mathrm{~K}$, relaxation time in Fig. 3 changes within one order of magnitude which is consistent with experimental observations 2, 15.

\section{CONCLUSIONS}

We have proposed a new defect-assisted mechanism of multiphonon intraband carrier relaxation in semiconductor QDs, where the carrier is found in a coherent superposition of the initial, final, and defect states. Combination of different approaches has helped us to devise a controlable approximation for the relaxation rate. We have shown that the proposed mechanism is capable of reproducing both decreasing, as it was observed for PbSe NCs 2, and increasing, as it was reported for CdSe QDs 15, temperature dependences of the relaxation time. For reasonable values of parameters, the change in the relaxation time with temperature rise from cryogenic to room temperatures was found within one order of magnitude, in agreement with experimental observations 2, 2.15 .

\section{Acknowledgments}

We are indebted to I.N. Yassievich for useful discussions and constant encouragement. This work was funded by the Russian Foundation for Basic Research under Grant 07-02-00469-a. A.N.P. acknowledges support by the Dynasty Foundation-ICFPM. 
1 J.M. Harbold, H. Du, T.D. Krauss, K.-S. Cho, C.B. Murray, and F.W. Wise, Phys. Rev. B 72, 195312 (2005).

2 R.D. Schaller, J.M. Pietryga, S.V. Goupalov, M.A. Petruska, S.A. Ivanov, and V.I. Klimov, Phys. Rev. Lett. 95, 196401 (2005).

3 Al.L. Efros, V.A. Kharchenko, and M. Rosen, Solid State Commun. 93, 281 (1995).

${ }^{4}$ K. Huang and A. Rhys, Proc. Roy. Soc. London A 204, 406 (1950); K. Huang, Scientia Sinica 24, 27 (1981).

5 B.K. Ridley, Quantum Processes in Semiconductors (Clarendon Press, Oxford, 1999). Ch. 6.

6 V.N. Abakumov, V.I. Perel, and I.N. Yassievich, Nonradiative recombination in semiconductors (North-Holland, Amsterdam 1991).

7 S.V. Goupalov, Phys. Rev. B 72, 073301 (2005).

8 X.-Q. Li and Y. Arakawa, Phys. Rev. B 56, 10423 (1997).

9 T.D. Krauss and F.W. Wise, Phys. Rev. B 55, 9860 (1997).

${ }^{10}$ I. Kang and F.V. Wise, J. Opt. Soc. Am. B 14, 1632 (1997).

11 P.C. Sercel, Phys. Rev. B 51, 14532 (1995).

12 D.F. Schroeter, D.J. Griffiths, and P.C. Sercel, Phys. Rev. B 54, 1486 (1996).

13 V.N. Abakumov, A.A. Pakhomov, and I.N. Yassievich, Pis'ma ZhETF 53, 167 (1991) [JETP Letters 53, 176
(1991)].

14 I.N. Yassievich, Semicond. Sci. Technol. 9, 1433 (1994).

15 P. Guyot-Sionnest, B. Wehrenberg, and D. Yu, J. Chem. Phys. 123, 074709 (2005).

16 L.I. Glazman and R.I. Shekhter, Zh. Exp. Teor. Fiz. 94, 292 (1988) [Sov. Phys. - JETP 67, 163 (1988)].

17 G.D. Mahan, Many-particle physics, 2nd edn (Plenum Press, N.Y. 1990). Sec. 4.3.

18 S.V. Goupalov, R.A. Suris, P. Lavallard, and D.S. Citrin, IEEE J. Selected Topics in Quantum Electronics 8, 1009 (2002).

19 It is convenient to think of $A(\omega)$ as of an optical absorption spectrum of a hypothetical system with optical gap at the frequency of the zero-phonon line.

20 A. Messiah, Quantum Mechanics (Dover, Mineola, N.Y. 1999).

21 V.B. Berestetskii, E.M. Lifshitz, and L.P. Pitaevskii, Quantum Electrodynamics (Pergamon Press, Oxford 1982) $\S 62$.

22 A.P. Prudnikov, Yu.A. Brychkov, and O.I. Marichev, Integrals and Series, Vol. 2, Special Functions, (Gordon \& Breach Sci. Publ., N.Y., 1986) Eq.2.20.10. 